\title{
On a singular elliptic problem involving critical growth in $\mathbb{R}^{\mathrm{N}}$
}

\author{
Manassés de Souza
}

\begin{abstract}
In this paper, we prove a suitable Trudinger-Moser inequality with a singular weight in $\mathbb{R}^{N}$ and as an application of this result, using the mountain-pass theorem we establish sufficient conditions for the existence of nontrivial solutions to quasilinear elliptic partial differential equations of the form

$$
-\Delta_{N} u+V(x)|u|^{N-2} u=\frac{f(x, u)}{|x|^{a}} \quad \text { in } \mathbb{R}^{N}, \quad N \geq 2,
$$

where $V: \mathbb{R}^{N} \rightarrow \mathbb{R}$ is a continuous potential, $a \in[0, N)$ and $f: \mathbb{R}^{N} \times \mathbb{R} \rightarrow$ $\mathbb{R}$ behaves like $\exp \left(\alpha|u|^{N /(N-1)}\right)$ when $|u| \rightarrow \infty$.
\end{abstract}

Mathematics Subject Classification (2000). 35J20, 35J60, 35B33.

Keywords. Mountain-pass theorem, Critical growth, Trudinger-Moser inequality.

\section{Introduction}

In this paper we deal with the existence of nontrivial solutions for the problem

$$
-\Delta_{N} u+V(x)|u|^{N-2} u=\frac{f(x, u)}{|x|^{a}} \quad \text { in } \mathbb{R}^{N}, \quad N \geq 2,
$$

where $\Delta_{N} u \equiv \operatorname{div}\left(|\nabla u|^{N-2} \nabla u\right)$ is the $N$-Laplacian and $a \in[0, N)$. Motivated by a Trudinger-Moser inequality with a singular weight (see Lemma 2.3 and $[22,28])$ we consider here the maximal growth on the nonlinear term $f(x, u)$ which allows to treat Eq. (1.1) variationally in a subspace of $W^{1, N}\left(\mathbb{R}^{N}\right)$, the Sobolev space of functions in $L^{N}\left(\mathbb{R}^{N}\right)$ such that its weak derivatives are also in $L^{N}\left(\mathbb{R}^{N}\right)$ with the norm

$$
\|u\|_{1, N} \doteq\left(\int_{\mathbb{R}^{N}}\left(|\nabla u|^{N}+|u|^{N}\right) \mathrm{d} x\right)^{1 / N}
$$


It is assumed that $f: \mathbb{R}^{N} \times \mathbb{R} \rightarrow \mathbb{R}$ is continuous, $f(x, 0) \equiv 0$ and $f$ behaves like $\exp \left(\alpha|u|^{N /(N-1)}\right)$ when $|u| \rightarrow \infty$. More precisely, we assume the following growth condition on the nonlinearity $f(x, u)$ :

$\left(f_{1}\right)$ There exist constants $\alpha_{0}, b_{1}, b_{2}>0$ such that for all $(x, u) \in \mathbb{R}^{N} \times \mathbb{R}$,

$$
|f(x, u)| \leq b_{1}|u|^{N-1}+b_{2} \Phi\left(\alpha_{0}|u|^{N /(N-1)}\right)
$$

where

$$
\Phi(t)=\exp (t)-\sum_{k=0}^{N-2} \frac{t^{j}}{j !} .
$$

The main features of this class of problems, considered in this paper, are the presence of the singularity $|x|^{-a}$, it is defined in the whole $\mathbb{R}^{N}$, involves critical growth and the nonlinear operator $N$-Laplacian. In spite of a possible failure of the Palais-Smale compactness condition, in this article we apply minimax methods, more precisely, the mountain-pass theorem, to obtain the existence of weak solutions of (1.1) in the subspace $E \subset W^{1, N}\left(\mathbb{R}^{N}\right)$ given by

$$
E=\left\{u \in W^{1, N}\left(\mathbb{R}^{N}\right): \int_{\mathbb{R}^{N}} V(x)|u|^{N} \mathrm{~d} x<\infty\right\} .
$$

We say that $u \in E$ is a weak solution of problem (1.1) if for all $v \in E$ we have

$$
\int_{\mathbb{R}^{N}}\left(|\nabla u|^{N-2} \nabla u \nabla v+V(x)|u|^{N-2} u v\right) \mathrm{d} x-\int_{\mathbb{R}^{N}} \frac{f(x, u)}{|x|^{a}} v \mathrm{~d} x=0 .
$$

Under the condition on the potential

$\left(V_{1}\right) V$ is a continuous function such that

$$
V(x) \geq V_{0}>0, \quad \text { for all } x \in \mathbb{R}^{N} .
$$

We can see that $E$ is a reflexive Banach space when endowed with the norm

$$
\|u\| \doteq\left(\int_{\mathbb{R}^{N}}\left(|\nabla u|^{N}+V(x)|u|^{N}\right) \mathrm{d} x\right)^{1 / N}
$$

and for all $N \leq q<\infty$,

$$
E \hookrightarrow W^{1, N}\left(\mathbb{R}^{N}\right) \hookrightarrow L^{q}\left(\mathbb{R}^{N}\right)
$$

with continuous embedding (see $[8,26]$ ). Moreover,

$$
\lambda_{1}(N) \doteq \inf _{u \in E \backslash\{0\}} \frac{\int_{\mathbb{R}^{N}}\left(|\nabla u|^{N}+V(x)|u|^{N}\right) \mathrm{d} x}{\int_{\mathbb{R}^{N}}|u|^{N} /|x|^{a} \mathrm{~d} x}>0 .
$$

We also assume suitable conditions on the potential $V$ in order to get the compactness of the following embedding

$$
E \hookrightarrow L^{p}\left(\mathbb{R}^{N}\right) \text { for all } p \geq N
$$

Notice that each one of the two conditions below are sufficient to get (1.4) (this fact can be found in $[8,20,24,26])$ :

$\left(V_{2}\right) V(x) \rightarrow \infty$ as $|x| \rightarrow \infty$; or more generally, for every $M>0$,

$$
\mu\left(\left\{x \in \mathbb{R}^{N}: V(x) \leq M\right\}\right)<\infty,
$$

with $\mu$ denoting the Lebesgue measure in $\mathbb{R}^{N}$; 
$\left(V_{3}\right) \int_{\mathbb{R}^{N}} V(x)^{-1} \mathrm{~d} x<\infty$.

We assume the following conditions on the nonlinearity $f(x, u)$ :

$\left(f_{2}\right)$ There exists $\theta>N$ such that for all $x \in \mathbb{R}^{N}$ and $u \geq 0$,

$$
0 \leq \theta F(x, u) \equiv \mu \int_{0}^{u} f(x, t) \mathrm{d} t \leq u f(x, u) .
$$

$\left(f_{3}\right)$ There exist constants $R_{0}, M_{0}>0$ such that, for all $x \in \mathbb{R}^{N}$ and $u \geq R_{0}$,

$$
0<F(x, u) \leq M_{0} f(x, u) .
$$

$\left(f_{4}\right) \limsup \sup _{u \rightarrow 0^{+}} \frac{N F(x, u)}{|u|^{N}}<\lambda_{1}(N)$ uniformly in $x \in \mathbb{R}^{N}$.

$\left(f_{5}\right) \lim _{u \rightarrow+\infty} u f(x, u) \exp \left(-\alpha_{0}|u|^{N /(N-1)}\right) \geq \beta_{0}>0$ uniformly on compact subsets of $\mathbb{R}^{N}$.

The main result of this paper is the following

Theorem 1.1. Suppose $\left(V_{1}\right)$ and $\left(V_{2}\right)$ (or $\left.\left(V_{3}\right)\right)$ and $\left(f_{1}\right)-\left(f_{5}\right)$ are satisfied. Then, the problem (1.1) has a nontrivial solution $u \in E$.

Remark 1.1. We point out that Theorem 1.1 is closely related with some results in [10] where the the existence and multiplicity of weak solutions for the following class of problems $-\Delta u+V(x) u=\frac{g(u)}{|x|^{a}}+h(x)$ in $\mathbb{R}^{2}$, where $a \in[0,2), V(x)$ is a continuous positive potential bounded away from zero and which can be "large" at the infinity, the nonlinearity $g(s)$ behaves like $e^{\alpha s^{2}}$ when $|s| \rightarrow+\infty$ and $h \in\left(H^{1}\left(\mathbb{R}^{2}\right)\right)^{*}$ is a small perturbation. See also [17] for others related results.

Remark 1.2. Notice that, in the semilinear case the hypotheses of Theorem 1.1 are for example satisfied by the nonlinearity

$$
f(x, u)=3 u^{2}+2 u \exp \left(u^{2}\right)-2 u .
$$

The condition $\left(f_{4}\right)$ is essential to guarantee our main results. For example, if $f(x, u)=\lambda_{1} u+\exp \left(u^{2}\right)-1+2 u^{2} \exp \left(u^{2}\right),(1.1)$ does not have positive solution.

Remark 1.3. It is well known that problems involving the $p$-Laplacian operator appear in many contexts. Some of these problems come from different areas of applied mathematics and physics. For example, they may be found in the study of non-Newtonian fluids, non-linear elasticity, and reaction-diffusions. For discussions about problems modelled by these boundary value problems, see for example [11].

Remark 1.4. The results in this paper were in part motivated by several recent papers on elliptic problems involving critical growth in the Trudinger-Moser case, see $[1,2,4,6,9,12,13,15,16,27]$ and references therein. Here we complement some the results mentioned above by establishing sufficient conditions for the existence of nontrivial solutions for singular case with $a \in[0, N)$. For problem (1.1), when $a \equiv 0$, the existence of nontrivial solutions has been studied on bounded domains by [9] in the semilinear case and by $[1,12,23]$ for the quasilinear equations. For problems in unbounded domains see $[6,13]$. 
This paper is organized as follows.

Section 2 we show a singular Trudinger-Moser inequality in $\mathbb{R}^{N}$ and contains some technical results that allows to give a variational approach of our work.

Section 3 contains the variational framework and we check the geometric conditions of the associated functional.

In Sect. 4 we get a more precise information about the minimax level obtained by the mountain-pass theorem.

Section 5 we prove our main results.

Throughout this paper we will choose subsequences of given sequences and relabeling them as the original sequence and we shall do without explicitly stating it.

$C, C_{1}, C_{2}, \ldots$ design positive generic constants and $B_{r}$ denotes a ball centered at the origin and radius $r$.

\section{A singular Trudinger-Moser inequality in $\mathbb{R}^{N}$}

Let $\Omega$ be a bounded domain in $\mathbb{R}^{N}$ with $N \geq 2$. The well known TrudingerMoser inequality (see $[22,28]$ ) asserts that

$$
\exp \left(\alpha|u|^{N /(N-1)}\right) \in L^{1}(\Omega), \quad \forall u \in W_{0}^{1, N}(\Omega), \forall \alpha>0 .
$$

Moreover, that there exists a constant $C=C(N)$, which depends only on $N$, such that

$$
\sup _{\|\nabla u\|_{N} \leq 1} \int_{\Omega} \exp \left(\alpha|u|^{N /(N-1)}\right) \mathrm{d} x \leq C(N)|\Omega|, \quad \text { if } \alpha \leq \alpha_{N},
$$

where $|\Omega|=\int_{\Omega} \mathrm{d} x, \alpha_{N}=N w_{N-1}^{1 /(N-1)}$ and $w_{N-1}$ is the $(N-1)$-dimensional measure of the $(N-1)$-sphere.

In the case of unbounded domains, we have the following version of the Trudinger-Moser inequality (see [13, Lemma 1], [6,23]).

Lemma 2.1. If $N \geq 2, \alpha>0$ and $u \in W^{1, N}\left(\mathbb{R}^{N}\right)$ then

$$
\int_{\mathbb{R}^{N}} \Phi\left(\alpha|u|^{N /(N-1)}\right) \mathrm{d} x<\infty .
$$

Moreover, if $\|\nabla u\|_{N}^{N} \leq 1,\|u\|_{N} \leq M<\infty$ and $\alpha<\alpha_{N}$ then there exists a constant $C=C(N, M, \alpha)$, which depends only on $N, M$ and $\alpha$, such that

$$
\int_{\mathbb{R}^{N}} \Phi\left(\alpha|u|^{N /(N-1)}\right) \mathrm{d} x \leq C(N, M, \alpha) .
$$

In a recent paper Adimurthi-Sandeep (see [3]) extended the TrudingerMoser inequality with a singular weight, more precisely:

Lemma 2.2. Let $\Omega$ be a bounded domain in $\mathbb{R}^{N}$ with $N \geq 2$, containing the origin and $u \in W_{0}^{1, N}(\Omega)$. Then for every $\alpha>0$ and $a \in[0, N)$

$$
\int_{\Omega} \frac{\exp \left(\alpha|u|^{N /(N-1)}\right)}{|x|^{a}} \mathrm{~d} x<\infty .
$$


Moreover,

$$
\sup _{\|\nabla u\|_{N} \leq 1} \int_{\Omega} \frac{\exp \left(\alpha|u|^{N /(N-1)}\right)}{|x|^{a}} \mathrm{~d} x<\infty,
$$

if and only if $\alpha / \alpha_{N}+a / N \leq 1$.

Next, inspired this last inequality and in [13, Lemma 1], we establish a version the Trudinger-Moser inequality with singular weight in $\mathbb{R}^{N}$. The proof of this result follows the same ideas in [17] and we omit it.

Lemma 2.3. If $\alpha>0, a \in[0, N)$ and $u \in W^{1, N}\left(\mathbb{R}^{N}\right)$ then

$$
\int_{\mathbb{R}^{N}} \frac{\Phi\left(\alpha|u|^{N /(N-1)}\right)}{|x|^{a}} \mathrm{~d} x<\infty .
$$

Moreover, if $\|\nabla u\|_{N} \leq 1,\|u\|_{N} \leq M<\infty$ and $\alpha / \alpha_{N}+a / N<1$. Then there exist a constant $C=C(N, M, \alpha)$, which depends only on $M$, and $\alpha$, such that

$$
\int_{\mathbb{R}^{N}} \frac{\Phi\left(\alpha|u|^{N /(N-1)}\right)}{|x|^{a}} \mathrm{~d} x \leq C(N, M, \alpha) .
$$

The next results are essential to establish the mountain-pass geometry of the associated functional.

Lemma 2.4. If $u \in E$ and $\|u\| \leq M$ with $\beta M^{N /(N-1)} / \alpha_{N}+a / N<1$ then

$$
\int_{\mathbb{R}^{N}} \frac{\Phi\left(\beta|u|^{N /(N-1)}\right)|u|}{|x|^{a}} \mathrm{~d} x \leq C(N, M, \beta)\|u\|_{s}
$$

for some $s>N$.

Proof. Using the Hölder inequality, we have

$$
\begin{aligned}
\int_{|x| \leq \sigma} \frac{\Phi\left(\beta|u|^{N /(N-1)}\right)|u|}{|x|^{a}} \mathrm{~d} x & \leq \int_{|x| \leq \sigma} \frac{\exp \left(\beta|u|^{N /(N-1)}\right)|u|}{|x|^{a}} \mathrm{~d} x \\
& \leq\left(\int_{|x| \leq \sigma} \frac{\exp \left(\beta r|u|^{N /(N-1)}\right)}{|x|^{a r}} \mathrm{~d} x\right)^{1 / r} \\
& \times\left(\int_{|x| \leq \sigma}|u|^{s} \mathrm{~d} x\right)^{1 / s}
\end{aligned}
$$

where $r>1$ is sufficiently close 1 such that $\beta r M^{N /(N-1)} / \alpha_{N}+a / N<1, s=$ $r /(r-1)>N$ and $\sigma$ is a number to be determined later. Now, using the Lemma 2.3, we obtain

$$
\int_{|x| \leq \sigma} \frac{\Phi\left(\beta|u|^{N /(N-1)}\right)|u|}{|x|^{a}} \mathrm{~d} x \leq C(\beta, N)\|u\|_{s} .
$$

Now, choosing $\sigma \geq 1$, we have

$$
\int_{|x| \geq \sigma} \frac{\Phi\left(\beta|u|^{N /(N-1)}\right)|u|}{|x|^{a}} \mathrm{~d} x \leq \int_{|x| \geq \sigma} \Phi\left(\beta|u|^{N /(N-1)}\right)|u| \mathrm{d} x,
$$


hence, by Lemma 2.2 in [14] there exist $C_{2}(N, M, \beta)>0$ such that

$$
\int_{|x| \geq \sigma} \frac{\Phi\left(\beta|u|^{N /(N-1)}\right)|u|}{|x|^{a}} \mathrm{~d} x \leq C_{2}(N, M, \beta)\|u\|_{s} .
$$

From (2.5) and (2.6), we obtain the Lemma.

Lemma 2.5. For $\beta / \alpha_{N}+a / N<1,\|u\| \leq M$ with $M$ sufficiently small and $q>N$, we have

$$
\int_{\mathbb{R}^{N}} \frac{\Phi\left(\beta|u|^{N /(N-1)}\right)|u|^{q}}{|x|^{a}} \mathrm{~d} x \leq C(\beta, N)\|u\|^{q} .
$$

Proof. The proof is analogous to the preceding proof.

\section{The variational framework}

We are interested in obtaining nonnegative weak solutions, it is convenient to define

$$
f(x, u)=0, \quad \forall(x, u) \in \mathbb{R}^{N} \times(-\infty, 0] .
$$

From $\left(f_{1}\right)$,we obtain for all $(x, u) \in \mathbb{R}^{N} \times \mathbb{R}$,

$$
|F(x, u)| \leq b_{3} \Phi\left(\alpha_{0}|u|^{N /(N-1)}\right)
$$

for some constant $b_{3}>0$. Thus, by Lemma 2.3, we have $F(x, u) /|x|^{a} \in L^{1}\left(\mathbb{R}^{N}\right)$ for all $u \in W^{1, N}\left(\mathbb{R}^{N}\right)$. Therefore, the functional $I: E \rightarrow \mathbb{R}$ given by

$$
I(u)=\frac{1}{N}\|u\|^{N}-\int_{\mathbb{R}^{N}} \frac{F(x, u)}{|x|^{a}} \mathrm{~d} x
$$

is well defined. Using the next proposition and standard arguments (see [5, Theorem A.VI] and [25, Appendix B]), we see that $I$ is a $C^{1}$ functional on $E$ with

$$
I^{\prime}(u) v=\int_{\mathbb{R}^{N}}\left(|\nabla u|^{N-2} \nabla u \nabla v+V(x)|u|^{N-2} u v\right) \mathrm{d} x-\int_{\mathbb{R}^{N}} \frac{f(x, u) v}{|x|^{a}} \mathrm{~d} x,
$$

for $v \in E$. Consequently, critical points of the functional $I$ are precisely the weak solutions of problem (1.1).

Proposition 3.1. Suppose that $\left(u_{n}\right)$ be a sequence in $W^{1, N}\left(\mathbb{R}^{N}\right)$ strongly convergent. Then there exist a subsequence $\left(u_{n_{k}}\right)$ of $\left(u_{n}\right)$ and $v \in W^{1, N}\left(\mathbb{R}^{N}\right)$ such that $\left|u_{n_{k}}(x)\right| \leq v(x)$ almost everywhere in $\mathbb{R}^{N}$.

Proof. See proposition 1 in [14].

Here, like in [26], we are going to use a mountain-pass theorem without a compactness condition such like the one of the Palais-Smale type. This version of mountain-pass theorem is a consequence of Ekeland's variational principle (see $[7,18,21])$. In the next two lemmas we check that the functional $I$ satisfies the geometric conditions of the mountain-pass theorem. 
Lemma 3.2. Suppose that $\left(V_{1}\right),\left(f_{1}\right)-\left(f_{5}\right)$ hold. Then there exists $\rho>0$ such that

$$
I(u)>0 \text { if }\|u\|=\rho .
$$

Proof. From $\left(f_{4}\right)$, there exist $\tau, \delta>0$ in such a way that $|u| \leq \delta$ implies

$$
F(x, u) \leq \frac{\left(\lambda_{1}(N)-\tau\right)}{N}|u|^{N},
$$

for all $x \in \mathbb{R}^{N}$. On the other hand, using $\left(f_{1}\right)$ for each $q>N$, there exists a constant $C=C(q, \delta)$ such that

$$
F(x, u) \leq C \Phi\left(\alpha_{0}|u|^{N /(N-1)}\right)|u|^{q},
$$

for $|u| \geq \delta$ and $x \in \mathbb{R}^{N}$. From (3.2) and (3.3) we obtain

$$
F(x, u) \leq \frac{\left(\lambda_{1}(N)-\tau\right)}{N}|u|^{N}+C \Phi\left(\alpha_{0}|u|^{N /(N-1)}\right)|u|^{q},
$$

for all $(x, u) \in \mathbb{R}^{N} \times \mathbb{R}$. Now, using Lemma 2.5, (1.3) and the continuous embedding $E \hookrightarrow L^{N}\left(\mathbb{R}^{N}\right)$, we obtain

$$
\begin{aligned}
I(u) & \geq \frac{1}{N}\|u\|^{N}-\frac{\left(\lambda_{1}(N)-\tau\right)}{N} \int_{\mathbb{R}^{N}} \frac{|u|^{N}}{|x|^{a}} \mathrm{~d} x-C\|u\|^{q} \\
& \geq \frac{1}{N}\left(1-\frac{\left(\lambda_{1}(N)-\tau\right)}{\lambda_{1}(N)}\right)\|u\|^{N}-C\|u\|^{q} .
\end{aligned}
$$

Hence

$$
I(u) \geq\|u\|\left[\frac{1}{N}\left(1-\frac{\left(\lambda_{1}(N)-\tau\right)}{\lambda_{1}(N)}\right)\|u\|^{N-1}-C\|u\|^{q-1}\right] .
$$

Since $\tau>0$ and $q>N$, we may choose $\rho>0$ such that

$$
\frac{1}{N}\left(1-\frac{\left(\lambda_{1}(N)-\tau\right)}{\lambda_{1}(N)}\right) \rho^{N-1}-C \rho^{q-1}>0 .
$$

Lemma 3.3. There exists $e \in E$ with $\|e\|>\rho$ such that

$$
I(e)<\inf _{\|u\|=\rho} I(u) .
$$

Proof. Let $u \in W^{1, N}\left(\mathbb{R}^{N}\right) \backslash\{0\}, u \geq 0$, with compact support $K=\operatorname{supp}(u)$. By $\left(f_{2}\right)$ and $\left(f_{3}\right)$ there exist $c>0$ and $d>0$ such that

$$
F(x, s) \geq c s^{\theta}-d, \quad \forall(x, s) \in K \times[0,+\infty) .
$$

Thus, since $\theta>N$, for $t>0$ we have

$$
I(t u) \leq \frac{t^{N}}{N}\|u\|^{N}-c t^{\theta} \int_{\mathbb{R}^{N}} \frac{u^{\theta}}{|x|^{a}} \mathrm{~d} x+d \int_{K} \frac{\mathrm{d} x}{|x|^{a}},
$$

which implies that $I(t u) \rightarrow-\infty$ as $t \rightarrow \infty$. Setting $e=t u$ with $t$ sufficiently large, the proof of the lemma follows. 


\section{The minimax level}

In order to get a more precise information about the minimax level obtained by the mountain-pass theorem, let us consider the following sequence of scaled and truncated Green's functions also considered by Moser (see [22]).

$$
\widetilde{M}_{n}(x, r)=\frac{1}{w_{N-1}^{1 / N}} \begin{cases}(\log n)^{(N-1) / N} & \text { if }|x| \leq r / n \\ \frac{\log \left(\frac{r}{|x|}\right)}{(\log n)^{1 / N}} & \text { if } r / n \leq|x| \leq r \\ 0 & \text { if }|x| \geq r .\end{cases}
$$

Notice that $\widetilde{M}_{n}(\cdot, r) \in W^{1, N}\left(\mathbb{R}^{N}\right)$, the support of $\widetilde{M}_{n}(x, r)$ is the ball $B_{r}$,

$$
\int_{\mathbb{R}^{N}}\left|\nabla \widetilde{M}_{n}(x, r)\right|^{N} \mathrm{~d} x=1 \quad \text { and } \quad \int_{\mathbb{R}^{N}}\left|\widetilde{M}_{n}(x, r)\right|^{N} \mathrm{~d} x=O(1 / \log n)
$$

as $n \rightarrow \infty$. Moreover, considering $M_{n}(x, r)=\widetilde{M}_{n}(x, r) /\left\|\widetilde{M}_{n}\right\|$, we can write

$$
M_{n}^{N /(N-1)}(x, r)=w_{N-1}^{-1 /(N-1)} \log n+d_{n}, \quad \forall|x| \leq r / n,
$$

where

$$
d_{n}=w_{N-1}^{-1 /(N-1)} \log n\left(\left\|\widetilde{M}_{n}\right\|^{-1 /(N-1)}-1\right) .
$$

Using (4.1), we conclude that $\left\|\widetilde{M}_{n}\right\| \rightarrow 1$ as $n \rightarrow \infty$. Consequently,

$$
\frac{d_{n}}{\log n} \rightarrow 0 \quad \text { as } \quad n \rightarrow \infty \text {. }
$$

Lemma 4.1. Let $\zeta_{n}=\left\|\widetilde{M}_{n}\right\|$, then

$$
\lim _{n \rightarrow+\infty} \zeta_{n} \log n \int_{0}^{\zeta_{n}^{-1}} \exp \left[(N-a) \log n\left(\xi^{N /(N-1)}-\zeta_{n} \xi\right)\right] \mathrm{d} \xi=\frac{N}{N-a} .
$$

Proof. Talking $t=\zeta_{n} \zeta$, we get

$$
\begin{aligned}
& \int_{0}^{\zeta_{n}^{-1}} \exp \left[(N-a) \log n\left(\xi^{N /(N-1)}-\zeta_{n} \xi\right)\right] \mathrm{d} \xi \\
& \quad=\zeta_{n}^{-1} \int_{0}^{1} \exp \left[(N-a) \log n\left[\left(t \zeta_{n}^{-1}\right)^{N /(N-1)}-t\right]\right] \mathrm{d} t
\end{aligned}
$$

Now, let $g:[0,1] \rightarrow \mathbb{R}$ the function defined by $g(t)=(N-a) \log$ $n\left[\left(t \zeta_{n}^{-1}\right)^{N /(N-1)}-t\right]$. Then

$$
g^{\prime}(0)=-(N-a) \log n
$$

and

$$
g^{\prime}(1)=(N-a) \log n\left(\frac{N}{N-1}\left(\zeta_{n}^{-1}\right)^{N /(N-1)}-1\right) .
$$

Let $\epsilon>0$ sufficiently small, then

$$
g(t)=-[(N-a) \log n] t+o(t), \quad t \in[0, \epsilon],
$$


and

$g(t)=(N-a) \log n\left(\frac{N}{N-1}\left(\zeta_{n}^{-1}\right)^{N /(N-1)}-1\right)(t-1)+o(t), \quad t \in[1-\epsilon, 1]$.

Therefore,

$$
\lim _{n \rightarrow \infty} \zeta_{n} \log n\left(\zeta_{n}^{-1} \int_{0}^{\epsilon} \exp \left[(N-a) \log n\left[\left(t \zeta_{n}^{-1}\right)^{N /(N-1)}-t\right]\right] \mathrm{d} t\right)=\frac{1}{N-a}
$$

and

$$
\lim _{n \rightarrow \infty} \zeta_{n} \log n\left(\zeta_{n}^{-1} \int_{1-\epsilon}^{1} \exp \left[(N-a) \log n\left[\left(t \zeta_{n}^{-1}\right)^{N /(N-1)}-t\right]\right] \mathrm{d} t\right)=\frac{N-1}{N-a} .
$$

On the other hand, we have

$$
\lim _{n \rightarrow \infty} \zeta_{n} \log n\left(\zeta_{n}^{-1} \int_{\epsilon}^{1-\epsilon} \exp \left[(N-a) \log n\left[\left(t \zeta_{n}^{-1}\right)^{N /(N-1)}-t\right]\right] \mathrm{d} t\right)=0 .
$$

From (4.4), (4.5) and (4.6), we obtain the result.

Lemma 4.2. Suppose that $\left(V_{1}\right)$ and $\left(f_{1}\right)-\left(f_{5}\right)$ hold. Then there exists $n \in \mathbb{N}$ such that

$$
\max _{t \geq 0}\left\{\frac{t^{N}}{N}-\int_{\mathbb{R}^{N}} \frac{F\left(x, t M_{n}\right)}{|x|^{a}} \mathrm{~d} x\right\}<\frac{1}{N}\left(\frac{N-a}{N} \frac{\alpha_{N}}{\alpha_{0}}\right)^{N-1} .
$$

Proof. Fix $r>0$ such that

$$
\beta_{0}>\frac{N-a}{N r^{N-a}}\left(\frac{N-a}{\alpha_{0}}\right)^{N-1}
$$

where $\beta_{0}$ has been given in the assumption $\left(f_{5}\right)$. Suppose, by contradiction, that for all $n$ we have

$$
\max _{t \geq 0}\left\{\frac{t^{N}}{N}-\int_{\mathbb{R}^{N}} \frac{F\left(x, t M_{n}\right)}{|x|^{a}} \mathrm{~d} x\right\} \geq \frac{1}{N}\left(\frac{N-a}{N} \frac{\alpha_{N}}{\alpha_{0}}\right)^{N-1},
$$

where $M_{n}(x)=M_{n}(x, r)$. In view of (3.5), for each $n$ there exists $t_{n}>0$ such that

$$
\frac{t_{n}^{N}}{N}-\int_{\mathbb{R}^{N}} \frac{F\left(x, t_{n} M_{n}\right)}{|x|^{a}} \mathrm{~d} x=\max _{t \geq 0}\left\{\frac{t^{N}}{N}-\int_{\mathbb{R}^{N}} \frac{F\left(x, t M_{n}\right)}{|x|^{a}} \mathrm{~d} x\right\} .
$$

Thus,

$$
\frac{t_{n}^{N}}{N}-\int_{\mathbb{R}^{N}} \frac{F\left(x, t_{n} M_{n}\right)}{|x|^{a}} \mathrm{~d} x \geq \frac{1}{N}\left(\frac{N-a}{N} \frac{\alpha_{N}}{\alpha_{0}}\right)^{N-1},
$$

and using the fact that $F(x, u) \geq 0$, we obtain

$$
t_{n}^{N} \geq\left(\frac{N-a}{N} \frac{\alpha_{N}}{\alpha_{0}}\right)^{N-1} .
$$


Since at $t=t_{n}$ we have

$$
\frac{d}{d t}\left(\frac{t^{N}}{N}-\int_{\mathbb{R}^{N}} \frac{F\left(x, t M_{n}\right)}{|x|^{a}} \mathrm{~d} x\right)=0,
$$

it follows that

$$
t_{n}^{N}=\int_{\mathbb{R}^{N}} \frac{t_{n} M_{n} f\left(x, t_{n} M_{n}\right)}{|x|^{a}} \mathrm{~d} x=\int_{|x| \leq r} \frac{t_{n} M_{n} f\left(x, t_{n} M_{n}\right)}{|x|^{a}} \mathrm{~d} x .
$$

Using hypothesis $\left(f_{5}\right)$, given $\tau>0$ there exists $R_{\tau}>0$ such that for all $u \geq R_{\tau}$ and $|x| \leq r$, we get

$$
u f(x, u) \geq\left(\beta_{0}-\tau\right) \exp \left(\alpha_{0}|u|^{N /(N-1)}\right) .
$$

From (4.9) and (4.10), for large $n$, we obtain

$$
\begin{aligned}
t_{n}^{N} & \geq\left(\beta_{0}-\tau\right) \int_{|x| \leq r / n} \frac{\exp \left(\alpha_{0}\left|t_{n} M_{n}\right|^{N /(N-1)}\right)}{|x|^{a}} \mathrm{~d} x \\
& =\left(\beta_{0}-\epsilon\right) \frac{w_{N-1}}{N-a}\left(\frac{r}{n}\right)^{N-a} \exp \left(\alpha_{0} t_{n}^{N /(N-1)} w_{N-1}^{-1 /(N-1)} \log n+\alpha_{0} t_{n}^{N /(N-1)} d_{n}\right) .
\end{aligned}
$$

Thus, setting

$$
H_{n} \doteq \frac{\alpha_{0} N \log n}{\alpha_{N}} t_{n}{ }^{N /(N-1)}+\alpha_{0} t_{n}{ }^{N /(N-1)} d_{n}-N \log t_{n}-(N-a) \log n
$$

we have

$$
1 \geq\left(\beta_{0}-\tau\right) \frac{w_{N-1}}{N-a} r^{N-a} \exp H_{n}
$$

Consequently, the sequence $\left(t_{n}\right)$ is bounded. Otherwise, up to subsequences, we would have

$$
\lim _{n \rightarrow \infty} H_{n}=+\infty,
$$

which leads to a contradiction. Moreover, by (4.3), (4.8) and

$$
\begin{aligned}
t_{n}^{N} \geq & \left(\beta_{0}-\tau\right) \frac{w_{N-1}}{N-a} r^{N-a} \\
& \times \exp \left\{\left[\frac{\alpha_{0} t_{n}{ }^{N /(N-1)}}{\alpha_{N}}-1\right](N-a) \log n+\alpha_{0} t_{n}^{N /(N-1)} d_{n}\right\},
\end{aligned}
$$

it follows that

$$
t_{n}^{N} \rightarrow\left(\frac{N-a}{N} \frac{\alpha_{N}}{\alpha_{0}}\right)^{N-1} \quad \text { as } n \rightarrow \infty .
$$

In order to estimate (4.9) more precisely, we consider the sets

$$
A_{n}=\left\{x \in B_{r}: t_{n} M_{n} \geq R_{\tau}\right\} \quad \text { and } \quad B_{n}=B_{r} \backslash A_{n} .
$$

From (4.9) and (4.10) we achieve

$$
\begin{aligned}
t_{n}^{N} \geq & \left(\beta_{0}-\tau\right) \int_{|x| \leq r} \frac{\exp \left(\alpha_{0}\left|t_{n} M_{n}\right|^{N /(N-1)}\right)}{|x|^{a}} \mathrm{~d} x+\int_{B_{n}} \frac{t_{n} M_{n} f\left(x, t_{n} M_{n}\right)}{|x|^{a}} \mathrm{~d} x \\
& -\left(\beta_{0}-\tau\right) \int_{B_{n}} \frac{\exp \left(\alpha_{0}\left|t_{n} M_{n}\right|^{N /(N-1)}\right)}{|x|^{a}} \mathrm{~d} x
\end{aligned}
$$


Notice that $M_{n}(x) \rightarrow 0$ and the characteristic functions $\chi_{B_{n}} \rightarrow 1$ for almost every $x$ such that $|x| \leq r$. Therefore, the Lebesgue's dominated convergence theorem implies

$$
\int_{B_{n}} \frac{t_{n} M_{n} f\left(x, t_{n} M_{n}\right)}{|x|^{a}} \mathrm{~d} x \rightarrow 0
$$

and

$$
\int_{B_{n}} \frac{\exp \left(\alpha_{0}\left|t_{n} M_{n}\right|^{N /(N-1)}\right)}{|x|^{a}} \mathrm{~d} x \rightarrow \frac{w_{N-1}}{N-a} r^{N-a} .
$$

Moreover, using that

$$
t_{n}^{N} \geq\left(\frac{N-a}{N} \frac{\alpha_{N}}{\alpha_{0}}\right)^{N-1}
$$

we have

$$
\begin{aligned}
& \int_{|x| \leq r} \frac{\exp \left(\alpha_{0}\left|t_{n} M_{n}\right|^{N /(N-1)}\right)}{|x|^{a}} \mathrm{~d} x \\
& \geq \int_{|x| \leq r} \frac{\exp \left(\frac{N-a}{N} \alpha_{N}\left|M_{n}\right|^{N /(N-1)}\right)}{|x|^{a}} \mathrm{~d} x \\
& =\int_{|x| \leq r / n} \frac{\exp \left(\frac{N-a}{N} \alpha_{N}\left|M_{n}\right|^{N /(N-1)}\right)}{|x|^{a}} \mathrm{~d} x \\
& \quad+\int_{r / n \leq|x| \leq r} \frac{\exp \left(\frac{N-a}{N} \alpha_{N}\left|M_{n}\right|^{N /(N-1)}\right)}{|x|^{a}} \mathrm{~d} x .
\end{aligned}
$$

and

$$
\begin{aligned}
& \int_{|x| \leq \frac{r}{n}} \frac{\exp \left(\frac{N-a}{N} \alpha_{N}\left|M_{n}\right|^{N /(N-1)}\right)}{|x|^{a}} \mathrm{~d} x \\
& =\int_{|x| \leq r / n} \frac{\exp \left[\frac{N-a}{N} \alpha_{N} \omega_{N-1}^{-1 /(N-1)} \log n+d_{n} \frac{N-a}{N} \alpha_{N}\right]}{|x|^{a}} \mathrm{~d} x \\
& =\frac{\omega_{N-1}}{N-a} \frac{r^{N-a}}{n^{N-a}} n^{\left[(N-a)+\frac{N-a}{N} \alpha_{N} \frac{d_{n}}{\log n}\right]} \rightarrow \frac{\omega_{N-1}}{N-a} r^{N-a},
\end{aligned}
$$

where we have used (4.3). Now, using the change of variable

$$
\xi=\frac{\log \frac{r}{s}}{\zeta_{n} \log n} \text { with } \zeta_{n}=\left\|\widetilde{M}_{n}\right\|,
$$

by straightforward computation, we have

$$
\begin{aligned}
& \int_{\frac{r}{n} \leq|x| \leq r} \frac{\exp \left(\frac{N-a}{N} \alpha_{N}\left|M_{n}\right|^{N /(N-1)}\right)}{|x|^{a}} \mathrm{~d} x \\
& =w_{N-1} r^{N-a} \zeta_{n} \log n \int_{0}^{\zeta_{n}^{-1}} \exp \left[(N-a) \log n\left(\xi^{N /(N-1)}-\zeta_{n} \xi\right)\right] \mathrm{d} \xi
\end{aligned}
$$


which converges to $\frac{w_{N-1}}{N-a} r^{N-a} N$ as $n \rightarrow \infty$, by Lemma 4.1. Finally, taking $n \rightarrow \infty$ in (4.12) and using (4.11) we obtain

$$
\left(\frac{N-a}{N} \frac{\alpha_{N}}{\alpha_{0}}\right)^{N-1} \geq\left(\beta_{0}-\tau\right) \frac{w_{N-1}}{N-a} r^{N-a} N,
$$

which implies that

$$
\beta_{0} \leq \frac{N-a}{N r^{N-a}}\left(\frac{N-a}{\alpha_{0}}\right)^{N-1},
$$

contrary to (4.7), and the proof is complete.

\section{Proof of Theorem 1.1}

In order to prove that a Palais-Smale sequence converges to a nontrivial solution of problem (1.1) we need to establish the following preliminary lemma.

Lemma 5.1. Let $\left(v_{n}\right) \subset E$ be a sequence such that $v_{n} \rightarrow u_{0}$ in $E$ and $\frac{f\left(x, v_{n}\right)}{|x|^{a}} \rightarrow \frac{f\left(x, u_{0}\right)}{|x|^{a}}$ in $L^{1}\left(B_{R}\right)$ for any $R>0$, then

$$
\frac{F\left(x, v_{n}\right)}{|x|^{a}} \rightarrow \frac{F\left(x, u_{0}\right)}{|x|^{a}} \text { in } L^{1}\left(\mathbb{R}^{N}\right) .
$$

Proof. For any $R>0$ we have

$$
\frac{f\left(x, v_{n}\right)}{|x|^{a}} \rightarrow \frac{f\left(x, u_{0}\right)}{|x|^{a}} \text { in } L^{1}\left(B_{R}\right) .
$$

Thus, there exists $g \in L^{1}\left(B_{R}\right)$ such that $\frac{\left|f\left(x, v_{n}\right)\right|}{|x|^{a}} \leq g$ almost everywhere in $B_{R}$. $\left(f_{3}\right)$ implies that $F\left(x, v_{n}\right) \leq M_{1}+M_{0} f\left(x, v_{n}\right)$ almost everywhere in $B_{R}$, where $M_{1}=\sup _{\left(x, v_{n}\right) \in B_{R} \times\left[0, R_{0}\right]} F\left(x, v_{n}\right)$. Thus, by generalized Lebesgue dominated convergence theorem (cf. [19])

$$
\frac{F\left(x, v_{n}\right)}{|x|^{a}} \rightarrow \frac{F\left(x, u_{0}\right)}{|x|^{a}} \text { in } L^{1}\left(B_{R}\right) .
$$

Setting $R \geq 1$ we have

$$
\int_{|x|>R} \frac{F\left(x, v_{n}\right)}{|x|^{a}} \mathrm{~d} x \leq \int_{|x|>R} F\left(x, v_{n}\right) \mathrm{d} x .
$$

Using $\left(f_{1}\right)$ and $\left(f_{2}\right)$, we have

$$
\begin{gathered}
\int_{|x|>R} F\left(x, v_{n}\right) \mathrm{d} x \leq C_{1} \int_{|x|>R}\left|v_{n}\right|^{N} \mathrm{~d} x \\
+C_{2} \int_{|x|>R}\left|v_{n}\right| \Phi\left(\alpha_{0}\left|v_{n}\right|^{N /(N-1))}\right) \mathrm{d} x
\end{gathered}
$$


and

$$
\begin{aligned}
\int_{|x|>R}\left|v_{n}\right| \Phi\left(\alpha_{0}\left|v_{n}\right|^{N /(N-1))}\right) \mathrm{d} x & =\sum_{j=N-1}^{\infty} \frac{\alpha_{0}^{j}}{j !} \int_{|x|>R}\left|v_{n}\right|\left|v_{n}\right|^{N j /(N-1)} \mathrm{d} x \\
& =\sum_{j=N-1}^{\infty} \frac{\alpha_{0}^{j}}{j !} \int_{|x|>R}\left|v_{n}^{*}\right|\left|v_{n}^{*}\right|^{N j /(N-1)} \mathrm{d} x
\end{aligned}
$$

where $v_{n}^{*}$ is the Schwarz symmetrization of $v_{n}$. Notice that the estimate

$$
\begin{aligned}
\int_{|x|>R} \frac{1}{|x|^{1+N j /(N-1)}} \mathrm{d} x & =w_{N-1} \int_{R}^{\infty} \frac{t^{N-1}}{t^{1+N j /(N-1)}} \mathrm{d} t \\
& =\left(\frac{w_{N-1}}{\frac{N j}{N-1}-N+1}\right) R^{N-1-N j /(N-1)} \leq \frac{w_{N-1}}{R},
\end{aligned}
$$

for all $j \geq N-1$, together with the Radial Lemma leads to

$$
\begin{aligned}
& \sum_{j=N-1}^{\infty} \frac{\alpha_{0}^{j}}{j !} \int_{|x|>R}\left|v_{n}^{*}\right|\left|v_{n}^{*}\right|^{N j /(N-1)} \mathrm{d} x \\
& \leq C\left(\frac{N}{w_{N-1}}\right)^{1 / N} \sum_{j=N-1}^{\infty} \frac{\alpha_{0}^{j}}{j !}\left(\frac{N}{w_{N-1}}\right)^{j /(N-1)} C^{N j /(N-1)} \\
& \int_{|x|>R}|x|^{-1-N j /(N-1)} \mathrm{d} x \\
& \leq \frac{C(N)}{R} .
\end{aligned}
$$

Thus, given $\delta>0$ there exists $R>0$ such that

$$
\int_{|x|>R}\left|u_{0}\right|^{N} \mathrm{~d} x<\delta \text { and } \int_{|x|>R}\left|v_{n}\right| \Phi\left(\alpha_{0}\left|v_{n}\right|^{N /(N-1)}\right) \mathrm{d} x<\delta,
$$

which together with (5.2) imply that

$$
\int_{|x|>R} F\left(x, v_{n}\right) \mathrm{d} x \leq C \delta \text { and } \int_{|x|>R} F\left(x, u_{0}\right) \mathrm{d} x \leq C \delta .
$$

Using the estimate

$$
\begin{aligned}
& \left|\int_{\mathbb{R}^{N}} \frac{F\left(x, v_{n}\right)}{|x|^{a}} \mathrm{~d} x-\int_{\mathbb{R}^{N}} \frac{F\left(x, u_{0}\right)}{|x|^{a}} \mathrm{~d} x\right| \\
& \leq\left|\int_{B_{R}} \frac{F\left(x, v_{n}\right)}{|x|^{a}} \mathrm{~d} x-\int_{B_{R}} \frac{F\left(x, u_{0}\right)}{|x|^{a}} \mathrm{~d} x\right| \\
& \quad+\int_{|x|>R} F\left(x, v_{n}\right) \mathrm{d} x+\int_{|x|>R} F\left(x, u_{0}\right) \mathrm{d} x
\end{aligned}
$$

we get

$$
\lim _{n \rightarrow \infty}\left|\int_{\mathbb{R}^{N}} \frac{F\left(x, v_{n}\right)}{|x|^{a}} \mathrm{~d} x-\int_{\mathbb{R}^{N}} \frac{F\left(x, u_{0}\right)}{|x|^{a}} \mathrm{~d} x\right| \leq C \delta,
$$

and since $\delta$ is arbitrary, the claim is proved. 
In view of Lemmas 3.2 and 3.3 we can apply the mountain-pass theorem without a compactness condition such like the one of the Palais-Smale type. (see $[7,18,21])$ to obtain a sequence $\left(u_{n}\right) \subset E$ such that $I\left(u_{n}\right) \rightarrow c>0$ and $I^{\prime}\left(u_{n}\right) \rightarrow 0$, that is,

$$
\begin{array}{r}
\frac{1}{N}\left\|u_{n}\right\|^{N}-\int_{\mathbb{R}^{N}} \frac{F\left(x, u_{n}\right)}{|x|^{a}} \mathrm{~d} x \rightarrow c \quad \text { as } n \rightarrow \infty, \\
\left|I^{\prime}\left(u_{n}\right) v\right| \leq \tau_{n}\|v\|, \quad \text { for all } v \in E,
\end{array}
$$

where $\tau_{n} \rightarrow 0$ as $n \rightarrow \infty$. Furthermore, by Lemma 4.2

$$
c<\frac{1}{N}\left(\frac{N-a}{N} \frac{\alpha_{N}}{\alpha_{0}}\right)^{N-1} .
$$

From now on, we shall be working in order to prove that $\left(u_{n}\right)$ converges to a weak nontrivial solution $u$ of problem (1.1). From (5.3), (5.4) and $\left(f_{2}\right)$, we get

$$
\begin{aligned}
C+\tau_{n}\left\|u_{n}\right\| & \geq\left(\frac{\theta}{N}-1\right)\left\|u_{n}\right\|^{N}-\int_{\mathbb{R}^{N}} \frac{\left(\theta F\left(x, u_{n}\right)-f\left(x, u_{n}\right) u_{n}\right)}{|x|^{a}} \mathrm{~d} x \\
& \geq\left(\frac{\theta}{N}-1\right)\left\|u_{n}\right\|^{N},
\end{aligned}
$$

which implies that

$$
\left\|u_{n}\right\| \leq C, \quad \int_{\mathbb{R}^{N}} \frac{f\left(x, u_{n}\right) u_{n}}{|x|^{a}} \mathrm{~d} x \leq C \quad \text { and } \quad \int_{\mathbb{R}^{N}} \frac{F\left(x, u_{n}\right)}{|x|^{a}} \mathrm{~d} x \leq C .
$$

Therefore, up to subsequences, we have $u_{n} \rightarrow u$ weakly in $E, u_{n} \rightarrow u$ in $L^{q}\left(\mathbb{R}^{N}\right), \forall q \geq N$ and $u_{n}(x) \rightarrow u(x)$ almost everywhere in $\mathbb{R}^{N}$. Moreover, using (5.5) and arguing as in Lemma 2.1 of [9] and Lemma 4 of [12] or Lemma 3.2 of [23], we get

$$
\left\{\begin{array}{l}
\frac{f\left(x, u_{n}\right)}{|x|^{a}} \rightarrow \frac{f(x, u)}{|x|^{a}} \text { in } L^{1}\left(B_{R}\right), \\
\left|\nabla u_{n}\right|^{N-2} \nabla u_{n} \rightarrow|\nabla u|^{N-2} \nabla u \text { weakly in }\left(L^{N /(N-1)}\left(B_{R}\right)\right)^{N},
\end{array}\right.
$$

for all $R>0$. Therefore by (5.4) passing to the limit, we have

$$
\int_{\mathbb{R}^{N}}\left(|\nabla u|^{N-2} \nabla u \nabla \varphi+V(x)|u|^{N-2} u \varphi\right) \mathrm{d} x-\int_{\mathbb{R}^{N}} \frac{f(x, u) \varphi}{|x|^{a}} \mathrm{~d} x=0
$$

for all $\varphi \in C_{0}^{\infty}\left(\mathbb{R}^{N}\right)$. Since $C_{0}^{\infty}\left(\mathbb{R}^{N}\right)$ is dense in $E$ then $u$ is a weak solution of (1.1). Let us show that $u$ is nontrivial. Assume, by contradiction, that $u \equiv 0$. By Lemma 5.1 we have

$$
\int_{\mathbb{R}^{N}} \frac{F\left(x, u_{n}\right)}{|x|^{a}} \mathrm{~d} x \rightarrow 0 \quad \text { as } n \rightarrow+\infty .
$$

This together with (5.3) imply

$$
\left\|u_{n}\right\|^{N} \rightarrow N c \quad \text { as } n \rightarrow+\infty
$$

and hence given $\epsilon>0$, we have $\left\|u_{n}\right\|^{N} \leq N c+\epsilon$, for large $n$. Using that

$$
c<\frac{1}{N}\left(\frac{N-a}{N} \frac{\alpha_{N}}{\alpha_{0}}\right)^{N-1}
$$


and choosing $q>1$ sufficiently close to 1 and $\epsilon$ sufficiently small, we obtain

$$
q \alpha_{0}\left\|u_{n}\right\|^{N /(N-1)} / \alpha_{N}+q a / N<1 .
$$

Hence, by Lemma 2.3, we have

$$
\int_{\mathbb{R}^{N}}\left(\frac{\Phi\left(\alpha\left|u_{n}\right|^{N /(N-1)}\right)}{|x|^{a}}\right)^{q} \mathrm{~d} x \leq C, \quad \text { for large } n,
$$

which, in combination with Hölder inequality and $\left(f_{1}\right)$, implies that

$$
\int_{\mathbb{R}^{N}} \frac{f\left(x, u_{n}\right)}{|x|^{a}} u_{n} \mathrm{~d} x \rightarrow 0 \quad \text { as } n \rightarrow+\infty .
$$

Therefore, from (5.4) with $v=u_{n}$, we achieve $\left\|u_{n}\right\| \rightarrow 0$ as $n \rightarrow+\infty$, which contradicts (5.7) since $c>0$. Thus, $u$ is nontrivial and the proof of our main result is complete.

\section{Acknowledgments}

This paper is a part of the author's Ph.D. thesis at the UFPE department of mathematics and the author is greatly indebted to his thesis adviser Professor João Marcos do Ó for many useful discussions, suggestions, and comments. The author also would like to thank the unknown referees for valuable comments and suggestions which improve the manuscript.

\section{References}

[1] Adimurthi, A.: Existence of positive solutions of the semilinear Dirichlet problem with critical growth for the $N$-Laplacian. Ann. Sc. Norm. Sup. Pisa 17, 393-413 (1990)

[2] Adimurthi, A., Yadava, S.L.: Critical exponent problem in $\mathbb{R}^{2}$ with Neumann boundary condition. Commun. Partial Differ. Equ. 15, 461-501 (1990)

[3] Adimurthi, A., Sandeep, K.: A singular Moser-Trudinger embedding and its applications. Nonlinear Differ. Equ. Appl. 13, 585-603 (2007)

[4] Alves, C.O., do Ó, J.M., Miyagaki, O.H.: On nonlinear perturbations of a periodic elliptic problem in $\mathbb{R}^{2}$ involving critical growth. Nonlinear Anal. 56, 781-791 (2004)

[5] Berestycki, H., Lions, P.L.: Nonlinear scalar field equations, I. Existence of ground state. Arch. Rat. Mech. Anal. 82, 313-346 (1983)

[6] Cao, D.M.: Nontrivial solution of semilinear elliptic equation with critical exponent in $\mathbb{R}^{2}$. Commun. Partial Diff. Equ. 17, 407-435 (1992)

[7] Chabrowski, J.: Variational methods for potential operator equations. With applications to nonlinear elliptic equations. In: de Gruyter Studies in Mathematics, vol. 24. Walter de Gruyter \& Co, Berlin (1997) 
[8] Costa, D.G.: On a class of elliptic systems in $\mathbb{R}^{N}$. Electron. J. Differ. Equ. (07), $1-14(1994)$

[9] de Figueiredo, D.G., Miyagaki, O.H., Ruf, B.: Elliptic equations in $\mathbb{R}^{2}$ with nonlinearities in the critical growth range. Calc. Var. 3, 139-153 (1995)

[10] de Souza, M., do Ó, J.M.: On singular Trudinger-Moser type inequality for unbounded domains in dimension two and its applications (preprint)

[11] Díaz, J.I.: Nonlinear partial differential equations and free boundaries. vol. I. Elliptic equations. In: Research Notes in Mathematics, vol. 106, 323 pp. Pitman, Boston (1985)

[12] do Ó, J.M.: Semilinear Dirichlet problems for the $N$-Laplacian in $\mathbb{R}^{N}$ with nonlinearities in critical growth range. Differ. Integral Equ. 9(5), 967-979 (1996)

[13] do Ó, J.M.: $N$-Laplacian equations in $\mathbb{R}^{N}$ with critical growth. Abstr. Appl. Anal 2(3-4), 301-315 (1997)

[14] do Ó, J.M., Medeiros, E., Severo, U.B.: On a quasilinear nonhomogeneous elliptic equation with critical growth in $\mathbb{R}^{N}$. J. Differ. Equ. 246, 1363-1386 (2009)

[15] do Ó, J.M., Souto, M.A.S.: On a class of nonlinear Schrödinger equations in $\mathbb{R}^{2}$ involving critical growth. J. Differ. Equ. 174, 289-311 (2001)

[16] do Ó, J.M., Ruf, B.: On a Schrödinger equation with periodic potential and critical growth in $\mathbb{R}^{2}$. NoDEA Nonlinear Differ. Equ. Appl. 13, 167-192 (2006)

[17] Giacomoni, J., Sreenadh, K.: A multiplicity result to a nonhomogeneous elliptic equation in whole space $\mathbb{R}^{2}$. Adv. Math. Sci. Appl. 15, 467-488 (2005)

[18] Ghoussoub, N.: Duality and Perturbation Methods in Critical Point Theory. Cambridge University Press, Cambridge (1993)

[19] Kavian, O.: Introduction la théorie des points critiques. Springer-Verlag France, Paris (1993)

[20] Kondratev, V., Shubin, M.: Discreteness of spectrum for the Schrödinger operators on manifolds of bounded geometry. Oper. Theory Adv. Appl. 110, 185-226 (1999)

[21] Mawhim, J., Willem, M.: Critical Point Theory and Hamiltonian Systems. Springer, Belin (1989)

[22] Moser, J.: A sharp form of an inequality by N. Trudinger. Ind. Univ. Math. J. 20, 1077-1092 (1971)

[23] Panda, R.: On semilinear Neumann problem with critical growth for the $n$-Laplacian. Nonlinear Anal. T.M.A. 26, 1347-1366 (1996)

[24] Omana, W., Willem, M.: Homoclinic orbits for a class of Hamiltonian systems. Differ. Integral Equ. 5, 1115-1120 (1992)

[25] Rabinowitz, P.H.: Minimax methods in critical point theory with applications to differential equations. CBMS Regional Conf. Ser. in Math., vol. 65 (1986) 
[26] Rabinowitz, P.H.: On a class of nonlinear Schrödinger equations. Z. Angew Math. Phys (Zamp) 43, 270-291 (1992)

[27] Silva, E., Soares, S.: Liouville-Gelfand type problems for the $N$-Laplacian on bounded domains of $\mathbb{R}^{N}$. Ann. Scuola Norm. Sup. Pisa Cl. Sci. 28(4), 1-30 (1999)

[28] Trudinger, N.S.: On the imbedding into Orlicz spaces and some applications. J. Math. Mech. 17, 473-484 (1967)

M. de Souza

Departamento de Matemática,

Universidade Federal de Pernambuco,

Recife, PE 50740-540, Brazil

e-mail: mxs@dmat.ufpe.br; manassesxavier@hotmail.com

Received: 16 Februay 2010.

Revised: 16 August 2010.

Accepted: 05 November 2010. 